No. of days
of
composting
0 (fresh
cuttings)
2
4
11
28
64

Interna]

temp. of Bacterial numbers (millions per gm. fresh compost

$20^{\circ} \mathrm{C}$. material)

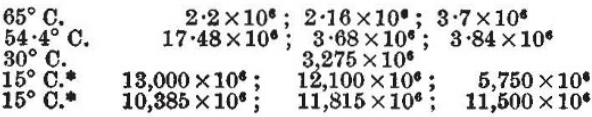

* Reached outside temperature

It will be seen that there is a reduction of the mesophilic bacterial flora present on the fresh material at the high-temperature phase $\left(65-55^{\circ} \mathrm{C}\right.$.). When the temperature of the heap falls below this, there is a very striking development of the mesophilic flora. The general picture of the bacteriology of this type of composting can best be shown by plotting the logarithm of the bacterial numbers against the days of composting.

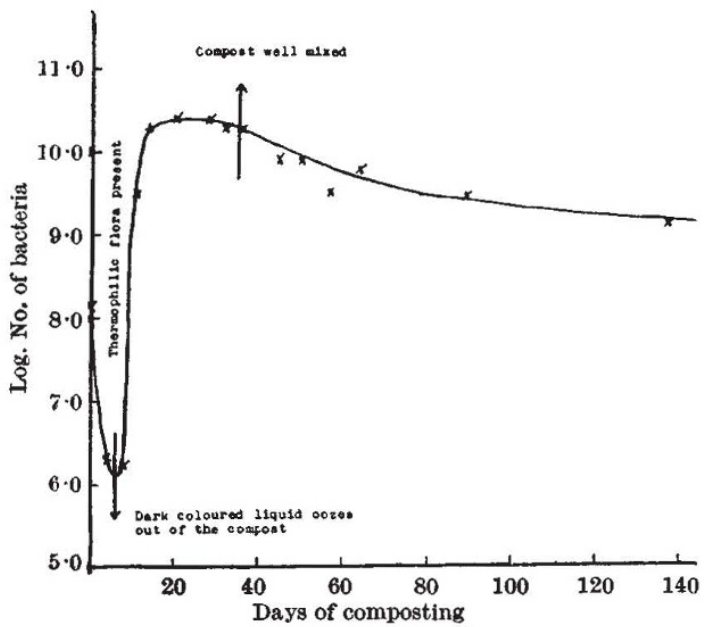

ChANGES IN THE AEROBIC BAOTERIAL FLORA (DEVELOPING ON NUTRIENT AGAR) DURING THE COMPOSTING OF GRASS CUTTINGS. CURVE REFERS TO MESOPHIIC BACTERIA

A similar course of events has been found with the mesophilic flora of a straw compost prepared following the principles of Hutchinson and Richards ${ }^{3}$. In the case of straw, however, no liquid appears during the composting (at least under our conditions), and the development of a thermophilic bacterial flora is not so obvious.

Macaulay Institute for Soil Research,

D. M. WEBLEY

Craigiebuckler,

Aberdeen.

Sept. 24.

${ }^{2}$ Webley, Nature, 159, 35 (1947).

Waksman, Umbreit and Cordon, Soil Sci., 47 (1), 37 (1939).

'Hutchinson and Richards, J. Min. Agric., 28, 398 (1921).

\section{Pre-erythrocytic Stage in Mammalian Malaria Parasites}

Shortt and Garnham have announced [Nature, January 24, p. 126] the discovery of plasmodial masses in the livers of two monkeys six and seven days respectively after inoculation with sporozoites of Plasmodium cynomolgi. These plasmodial masses apparently form the long-sought pre-erythrocytic stage of mammalian malaria parasites, and it is to be expected that human malaria parasites will show similar developmental changes in the incubation period of an infection. Prof. Shortt has very kindly shown me his preparations, and I write to confirm his discovery by the finding of similar parasites in my own laboratory.

For several years we have studied the life-cycle of avian and simian malaria parasites, and biological information has been collected about development of $P$. cynomolgi. After the injection of sporozoites into the skin of monkeys, sporozoites have been demonstrated microscopically at the site of injection for $4 \frac{1}{3}$ hours but not longer; these sporozoites lie extracellularly and probably represent forms which fail to develop further. By subinoculation, sporozoites have been demonstrated in the blood of a limb (in which circulation had been arrested by a tourniquet) as late as two hours after intradermal injection. Following this initial period (as is well known) the blood ceases to be infective on subinoculation for approximately eight days. The time at which infectivity returns is sharply defined. Monkeys inoculated with blood taken at 7 days 21 hours, at 7 days 15 hours, and at many shorter periods never became infected; monkeys inoculated with blood taken at 7 days 23 hours, 8 days 4 hours, or longer periods did become infected. Meticulous examination of blood films between the eighth and thirteenth day of the infection showed that the entry of parasites into the blood stream was not synchronous but that it was a continuous process spreading over several days. Subinoculation of tissues (including liver) from monkeys during the inoculation period was done repeatedly; but no transmission was ever achieved with tissue removed less than 7 days 23 hours after the injection of sporozoites. The tissue forms were not transmissible by this means.

Our attempts to locate and identify the tissue forms were unsuccessful until Prof. Shortt's kind demonstration of this preparation. On re-examination of our sections, forms similar to those he has described have been found in the livers of three monkeys. In one experiment, a three months old rhesus monkey was inoculated intravenously and parenterally with the glands from 120 infected mosquitoes. It was killed 7 days 23 hours later. The blood, liver, lung and spleen respectively were infective to other monkeys, but other organs did not produce infection. Large plasmodial masses (average diameter about $28 \mu$ ) have been found in the liver; this monkey had been bred in the laboratory, so there could be no question of some other natural infection acquired in India. Similar forms have been found in the liver of a monkey killed 7 days 15 hours after the intravenous injection of the ground-up thoraces of 1,000 infected mosquitoes; the blood and organs were not infective for other monkeys. A third monkey was inoculated intravenously with a suspension of 40 infected mosquitoes (ground up), and it was killed exactly five days later. The blood, liver and other organs did not infect monkeys into which they were sub-inoculated, but parasites (average of longest diameter $14 \mu$ ) have been found in the parenchymatous cells of the liver.

In all these cases, parasites are much more scanty and difficult to find in smears than in sections. Further investigation is proceeding.

Frank Hawking

National Institute for Medical Research, London, N.W.3.

Jan. 22. 